\title{
Clustering of lifestyle factors and association with overweight in adolescents of the Kiel Obesity Prevention Study
}

\author{
Beate Landsberg, Sandra Plachta-Danielzik, Dominique Lange, Maike Johannsen, \\ Jasmin Seiberl and Manfred James Müller* \\ Institut für Humanernährung und Lebensmittelkunde, Christian-Albrechts-Universität zu Kiel, Düsternbrooker Weg \\ 17, D-24105 Kiel, Germany
}

Submitted February 2009: Accepted May 2010

\begin{abstract}
Objective: To identify lifestyle clusters in adolescents and to characterize their association with overweight and obesity.

Design: Cross-sectional and longitudinal data of the Kiel Obesity Prevention Study.

Setting: Schools in Kiel, Germany.

Subjects and methods: Cross-sectional data of 1894 adolescents aged 14 years and 4-year longitudinal data of a subsample of 389 children aged 10 and 14 years. Selfreported data of physical activity, modes of commuting to school, media time, nutrition, alcohol consumption and smoking were used to identify lifestyle clusters with two-step cluster analysis. Obesity indices (height, weight, waist circumference and fat mass (FM)) were measured.

Results: Three lifestyle clusters were identified: a 'low activity and low-risk behaviour' cluster (cluster 1: $n 740,39 \cdot 1 \%$ ); a 'high media time and high-risk behaviour' cluster (cluster 2: $n$ 498, 26.3\%); and a 'high activity and medium-risk behaviour' cluster (cluster 3: $n$ 656, 34.6\%). Strictly speaking, none of these clusters was considered to be markedly healthy. The prevalence of overweight and obesity tended to be lower in cluster $3(15 \cdot 9 \%)$ than in clusters $1(20 \cdot 4 \%)$ and $2(20.5 \% ; P=0.053)$. Longitudinally, 4-year changes in FM were found to be lowest in cluster 2, but the 4-year incidence rate of obesity was lowest in cluster 3.

Conclusions: Explicit healthy lifestyles do not exist, but an active lifestyle reduces the incidence of obesity. In adolescents, health promotion should take into account the diversity of lifestyles and address specific lifestyle clusters.
\end{abstract}

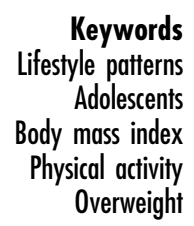

Lifestyle factors such as high television (TV) viewing ${ }^{(1-3)}$, sugar-sweetened beverage consumption ${ }^{(4-6)}$ and excess snacking ${ }^{(7)}$ are associated with increased obesity risk while high physical activity $(\mathrm{PA})^{(8-10)}$ and longer sleep duration $^{(3,11,12)}$ appear to be protective. However, in most studies these behaviours account for a minor proportion of the variance of overweight and obesity. Moreover, usually only one or selected lifestyle factors have been explored. However, individual lifestyle factors tend to cluster among themselves. For example, snack frequency and sugarsweetened beverage consumption increase with the hours of TV viewing ${ }^{(13,14)}$ while sedentary behaviours and physical activities co-occur ${ }^{(15-17)}$. A combination of health behaviours may have synergistic effects on overweight. Only a few studies have addressed the clustering of lifestyle factors. Understanding the interrelationships among lifestyle factors will provide insights for health research and for development of public health programmes that can impact on the obesity epidemic.
Within the Kiel Obesity Prevention Study (KOPS) ${ }^{(18,19)}$, we conducted a cross-sectional study in German eighth-grade students to identify lifestyle clusters composed of PA, commuting mode, media time, nutrition, alcohol consumption and smoking. These clusters were related to obesity indices. In addition, a longitudinal study was performed to investigate the impact of these clusters on the incidence of overweight and obesity.

\section{Methods}

\section{Study population}

Data were collected as part of KOPS, which is an ongoing study on the determinants and on the effect of the prevention of childhood overweight started in 1996 in Kiel, Germany ${ }^{(18,19)}$. In KOPS, three groups of children and adolescents were enrolled: (i) 6-year-old children ( $n$ 4997; as part of a school entry examination in Kiel between 1996 
and 2001); (ii) 10-year-old children ( $n$ 4487; within a school examination between 2000 and 2005); and (iii) 14-year-old adolescents ( $n$ 3237; as part of secondary schools in Kiel between 2004 and 2006). The heads of all thirty-five secondary schools in Kiel were contacted and informed, of which three refused to participate. All eighth-grade students ( $n$ 6783) of the remaining schools were invited to attend the study. Participation was on a voluntary basis and there were no eligibility criteria except the willingness to participate. In total, $48 \%$ ( $n$ 3237) of the students agreed to take part in the investigation. No information is available regarding the 3546 students who did not participate in the study. Signed informed consent was obtained and the study protocol was approved by the local ethics committee.

Of the 3237 adolescents, 1343 had missing data on lifestyle factors ( $n$ 1320) or obesity indices ( $n$ 23). Thus, the 'study population' of the present study consists of 1894 14-year-old adolescents of the third KOPS group. Since all three study groups of KOPS belonged to the same total population, a 'subsample' of 389 adolescents was identified whose obesity indices had been measured twice within a 4-year follow-up period (at the age of 10 (T0) and 14 (T1) years).

\section{Lifestyle factors}

\section{Physical activity and media time}

PA, commuting mode to school and media time were assessed with a questionnaire. Weekly duration of regular structured PA outside school as well as weekly duration of unstructured PA (e.g. jogging and skating) was provided by the adolescents. Four-week test-retest correlation $(r)$ was $0.50(P<0.01)$ and $0.35(P<0 \cdot 01)$ for the duration of structured and unstructured PA, respectively ${ }^{(20)}$. Mode of commuting to school was given and categorized as active (walking and cycling) and inactive (driving by car or bus) commuting. Test-retest percentage agreement (reliability) was $91.6 \%$. Self-reported media time was assessed as hours per day spent in TV viewing and computer use on a typical weekday. The 4-week test-retest correlation $(r)$ was $0.68(P<0 \cdot 01)$. In a previous study on $5-11$-year-old children, TV viewing had been compared with (i) energy expenditure as assessed by the combined use of indirect calorimetry together with $24 \mathrm{~h}$ heart rate monitoring (time more than Flex heart rate); and (ii) aerobic fitness (submaximal oxygen consumption, $\mathrm{O}_{2}$ pulse) ${ }^{(21)}$. However, there were no significant differences in either energy expenditure or fitness between groups of children watching $\mathrm{TV} \leq 1 \mathrm{~h} v .>1 \mathrm{~h} / \mathrm{d}$.

\section{Nutrition and risk behaviour}

A twenty-six-item validated FFQ was used to describe dietary intake ${ }^{(22)}$. Consumption of three or more 'healthy' foods (whole meal bread, fruits, vegetables, fish, dairy products) and fewer than three 'risk-related' foods (white bread, sausage, soft drinks, fast food, sweets/chips) at least 3-5 times a week was summarized as a 'healthy dietary pattern'(20). Consumption of three or more 'riskrelated' foods and fewer than three 'healthy' foods at least 3-5 times a week corresponded to a 'risk-related dietary pattern'. Other combinations indicated a 'mixed dietary pattern'. The 4-week test-retest percentage agreement (reliability) of dietary pattern was $67 \cdot 6 \%$.

Risk behaviour was defined as alcohol consumption and smoking behaviour. Habitual alcohol consumption was assessed according to: never, once a month, 2-3 times a month and at least once a week. For the analysis, the latter three categories were combined. Adolescents were also asked whether they smoked or not. The 4 -week test-retest percentage agreement (reliability) was $78.3 \%$ for alcohol consumption and $95.6 \%$ for smoking.

\section{Obesity indices and blood pressure}

Obesity indices were measured anthropometrically (height, weight, waist circumference (WC)) and bioelectric impedance analysis (fat mass (FM) calculated with a populationspecific algorithm $)^{(18,19,23)}$ was performed at the age of 10 and 14 years. International age- and gender-specific cutoffs were used to define overweight and obesity by BMI ${ }^{(24)}$. German references were used to calculate the SD score of $\mathrm{BMI}^{(25)}$. Blood pressure was measured using a sphygmomanometer on the right arm after a 5-min rest.

\section{Social characteristics}

Educational level of the adolescents was defined using the attended school type. Germany's school system is divided into three different types of school: 'Hauptschule' (nine school-years), 'Realschule' (ten school-years) and 'Gymnasium' (at least twelve school-years) depending on the level of academic performance of the students. In the present study, educational level was expected to be 'low' in the 'Hauptschule', 'middle' in the 'Realschule' and 'high' in the 'Gymnasium'. The nationality of the adolescents and their families was acquired and transformed into a dichotomous variable (German $v$. non-German).

\section{Statistical analysis}

Results were presented as median and interquartile range. A non-parametric Kruskal-Wallis test was used to determine between-group differences with respect to continuous variables. The $\chi^{2}$ test compared prevalence of categorical variables among groups.

\section{Lifestyle clusters}

Two-step cluster analysis was used (the Statistical Package for the Social Sciences statistical software package version $16 \cdot 0$ (SPSS Inc., Chicago, IL, USA)) as an explanatory tool to identify specific lifestyle patterns. This method is designed to handle large data sets and enables the input of both categorical and continuous variables ${ }^{(26)}$. The categorical variables included commuting mode to 
Table 1 Obesity indices and lifestyle factors in the study population of 14-year-old adolescents $(n 1894)$ and in the subsample of 389 subjects at the age of 10 (T0) and 14 (T1) years: Kiel Obesity Prevention Study, 2000-2006

\begin{tabular}{|c|c|c|c|c|c|c|}
\hline & & & \multicolumn{4}{|c|}{ Subsample } \\
\hline & \multicolumn{2}{|c|}{ Study population } & \multicolumn{2}{|r|}{ TO } & \multicolumn{2}{|r|}{ T1 } \\
\hline & Median & IQR & Median & IQR & Median & IQR \\
\hline Age (years) & $14 \cdot 7$ & $14 \cdot 3-15 \cdot 1$ & $10 \cdot 2$ & $9 \cdot 9-10 \cdot 6$ & $14 \cdot 6$ & $14 \cdot 3-15 \cdot 1$ \\
\hline \multicolumn{7}{|l|}{ Obesity indices } \\
\hline Weight (kg) & $58 \cdot 2$ & $52 \cdot 1-65 \cdot 7$ & $36 \cdot 0$ & $31 \cdot 8-41 \cdot 1$ & $59 \cdot 0$ & $52 \cdot 3-65 \cdot 4$ \\
\hline Height $(m)$ & $1 \cdot 68$ & $1 \cdot 63-1 \cdot 74$ & $1 \cdot 44$ & $1 \cdot 39-1 \cdot 48$ & $1 \cdot 69$ & $1 \cdot 63-1 \cdot 74$ \\
\hline BMI $\left(\mathrm{kg} / \mathrm{m}^{2}\right)$ & $20 \cdot 4$ & $18 \cdot 8-22 \cdot 6$ & $17 \cdot 2$ & $16 \cdot 1-19 \cdot 1$ & $20 \cdot 3$ & $19 \cdot 0-22 \cdot 5$ \\
\hline BMI-SDS & $0 \cdot 23$ & $-0 \cdot 37-0 \cdot 87$ & $0 \cdot 12$ & $-0.40-0.86$ & 0.23 & $-0 \cdot 26-0 \cdot 85$ \\
\hline FM (\%) & $22 \cdot 6$ & $16 \cdot 7-27 \cdot 9$ & $20 \cdot 8$ & $16 \cdot 7-26 \cdot 2$ & $22 \cdot 3$ & $16 \cdot 5-28 \cdot 4$ \\
\hline FM Z-score & $-0 \cdot 15$ & $-0 \cdot 68-0.55$ & $0 \cdot 10$ & $-0.51-0.93$ & -0.09 & $-0.66-0.64$ \\
\hline $\mathrm{WC}(\mathrm{cm})$ & $69 \cdot 3$ & $65 \cdot 5-74 \cdot 2$ & $63 \cdot 0$ & $59 \cdot 5-67 \cdot 5$ & $70 \cdot 0$ & $66 \cdot 0-74 \cdot 0$ \\
\hline Prevalence of overweight (\%) & \multicolumn{2}{|r|}{$14 \cdot 5$} & \multicolumn{2}{|r|}{$16 \cdot 2$} & \multicolumn{2}{|r|}{$12 \cdot 9$} \\
\hline Prevalence of obesity (\%) & \multicolumn{2}{|r|}{$4 \cdot 4$} & \multicolumn{2}{|c|}{$2 \cdot 8$} & \multicolumn{2}{|r|}{$4 \cdot 4$} \\
\hline \multicolumn{7}{|l|}{ Lifestyle factors } \\
\hline Duration of structured PA (h/week) & $2 \cdot 0$ & $0.0-4.5$ & $2 \cdot 0$ & $0 \cdot 0-4 \cdot 0$ & $2 \cdot 0$ & $0.0-4.5$ \\
\hline Duration of unstructured PA (h/week) & $2 \cdot 5$ & $1 \cdot 3-5 \cdot 0$ & & - & $2 \cdot 5$ & $1 \cdot 0-5 \cdot 0$ \\
\hline \multicolumn{7}{|l|}{ Commuting to school (\%) } \\
\hline Actively & \multicolumn{2}{|r|}{$72 \cdot 1$} & \multicolumn{2}{|r|}{ - } & \multicolumn{2}{|r|}{$78 \cdot 9$} \\
\hline Inactively & \multicolumn{2}{|r|}{$27 \cdot 9$} & \multicolumn{2}{|r|}{ - } & \multicolumn{2}{|r|}{$21 \cdot 1$} \\
\hline Media time (h/day) & $2 \cdot 5$ & $1 \cdot 5-3 \cdot 5$ & $1 \cdot 5$ & $1 \cdot 0-2 \cdot 0$ & $2 \cdot 5$ & $2 \cdot 0-3 \cdot 5$ \\
\hline \multicolumn{7}{|l|}{ Dietary pattern (\%) } \\
\hline Healthy & \multicolumn{2}{|r|}{$29 \cdot 4$} & \multicolumn{2}{|c|}{$5 \cdot 7$} & \multicolumn{2}{|r|}{$26 \cdot 7$} \\
\hline Risk-related & \multicolumn{2}{|r|}{$15 \cdot 5$} & \multicolumn{2}{|c|}{$24 \cdot 1$} & \multicolumn{2}{|r|}{$18 \cdot 5$} \\
\hline Mixed & \multicolumn{2}{|r|}{$55 \cdot 1$} & \multicolumn{2}{|c|}{$70 \cdot 2$} & \multicolumn{2}{|r|}{$54 \cdot 8$} \\
\hline \multicolumn{7}{|l|}{ Alcohol consumption (\%) } \\
\hline Never & \multicolumn{2}{|r|}{$59 \cdot 9$} & & - & & $59 \cdot 6$ \\
\hline At least once a month & & $40 \cdot 1$ & & - & & $40 \cdot 4$ \\
\hline Smokers (\%) & & $12 \cdot 7$ & & - & & $11 \cdot 8$ \\
\hline
\end{tabular}

IQR, interquartile range; BMI-SDS, SD score of BMI; FM, fat mass; WC, waist circumference; PA, physical activity.

school, dietary pattern, alcohol consumption and smoking behaviour. The continuous variables included duration of structured and unstructured PA and media time. The choice of similarity measure and the determination of the number of clusters were based on the log-likelihood distance and Schwarz's Bayesian criterion. To demonstrate the robustness of the cluster solution, the solution was re-examined on an internal random sample of $50 \%$ of the study population. The different clusters are given labels that characterize the behavioural pattern. The identified clusters were then compared regarding the actual difference on the lifestyle behaviours by means of ANOVA and a Bonferroni post hoc test. Multivariate logistic regression analyses were performed to estimate OR for overweight and obesity.

\section{Longitudinal data}

Obesity indices of the subsample of 389 adolescents were measured twice assuming an identical lifestyle pattern during the follow-up period (T1-T0). The follow-up period was 4.5 (SD 0.4 ) years. T1 data were corrected to 4 years by linear extrapolation using the following equation: $\mathrm{BMI}_{\text {corrected }}(\mathrm{T} 1)=[(\mathrm{BMI}(\mathrm{T} 1)-\mathrm{BMI}(\mathrm{T} 0)] /[$ age (T1)-age $(\mathrm{T} 0) \times 4]+$ BMI $(\mathrm{T} 0)$.

All analyses were performed with SPSS version $16 \cdot 0$ and the level of significance was set at $P<0.05$ (two-sided).

\section{Results}

\section{Characteristics of the study population and the subsample}

The study population consisted of 1894 14-year-old adolescents ( $48.5 \%$ boys). Most of the adolescents had high and middle educational levels ( $41.6 \%$ and $38.9 \%$, respectively). Non-German adolescents made up $14 \%$ of the total. When compared with the study population, the percentage of boys was higher $(52 \cdot 4 \%)$ and the percentage of non-Germans was lower $(7 \cdot 5 \%)$ in the subsample of 389 subjects. Obesity indices and lifestyle factors in the study population and the subsample are shown in Table 1.

\section{Cross-sectional data}

Characterization of lifestyle clusters

The present study identified three lifestyle clusters. Based on the cluster criteria, cluster 1 was characterized by 'low activity and low-risk behaviour', cluster 2 by 'high media time and high-risk behaviour' and cluster 3 by 'high activity and medium-risk behaviour' (Table 2). A generally healthy lifestyle pattern could not be identified in our study population.

There were $39.1 \%$ of adolescents who were affiliated to cluster 1 . This cluster was characterized by low-structured and unstructured PA and moderate media time. Of the adolescents, one-fifth had a risk-related dietary pattern. 
Table 2 Cluster centres for the lifestyle factors included in the cluster analyses: cross-sectional data, Kiel Obesity Prevention Study, 2004-2006

\begin{tabular}{|c|c|c|c|c|c|c|c|c|c|}
\hline & \multicolumn{3}{|c|}{ Cluster 1} & \multicolumn{3}{|c|}{ Cluster 2} & \multicolumn{3}{|c|}{ Cluster 3} \\
\hline & \multicolumn{3}{|c|}{$\begin{array}{l}\text { Low activity and low-risk } \\
\text { behaviour }(n 740)\end{array}$} & \multicolumn{3}{|c|}{$\begin{array}{c}\text { High media time and high-risk } \\
\text { behaviour ( } n \text { 498) }\end{array}$} & \multicolumn{3}{|c|}{$\begin{array}{l}\text { High activity and medium-risk } \\
\text { behaviour ( } n \text { 656) }\end{array}$} \\
\hline & Mean & SD & Z-score & Mean & SD & Z-score & Mean & SD & Z-score \\
\hline $\begin{array}{l}\text { Duration of structured PA (h/week) } \\
\text { Duration of unstructured PA (h/week) } \\
\text { Media time (h/d) }\end{array}$ & $\begin{array}{l}2 \cdot 4^{\mathrm{a}} \\
3 \cdot 3^{\mathrm{a}} \\
2 \cdot 8^{\mathrm{a}}\end{array}$ & $\begin{array}{l}2 \cdot 8 \\
4 \cdot 2 \\
1 \cdot 6\end{array}$ & $\begin{array}{r}-0 \cdot 18 \\
-0 \cdot 16 \\
0.05\end{array}$ & $\begin{array}{l}2 \cdot 7^{\mathrm{a}} \\
4 \cdot 2^{\mathrm{b}} \\
3 \cdot 1^{\mathrm{b}}\end{array}$ & $\begin{array}{l}3 \cdot 6 \\
4 \cdot 5 \\
1 \cdot 6\end{array}$ & $\begin{array}{r}-0.09 \\
0.03 \\
0.21\end{array}$ & $\begin{array}{l}3 \cdot 7^{b} \\
4 \cdot 6^{b} \\
2 \cdot 2^{c}\end{array}$ & $\begin{array}{l}3 \cdot 9 \\
5 \cdot 8 \\
1 \cdot 4\end{array}$ & $\begin{array}{r}0 \cdot 28 \\
0 \cdot 16 \\
-0 \cdot 22\end{array}$ \\
\hline Media time $(\mathrm{h} / \mathrm{d})$ & & $\%$ & & & $\%$ & & & $\%$ & \\
\hline \multicolumn{10}{|l|}{ Commuting to school } \\
\hline Actively & & $73 \cdot 1$ & & & $69 \cdot 1$ & & & $73 \cdot 3$ & \\
\hline \multicolumn{10}{|l|}{ Dietary pattern } \\
\hline Healthy & & $25 \cdot 5 t$ & & & $21 \cdot 3 t$ & & & $39 \cdot 9+$ & \\
\hline Risk-related & & $19 \cdot 1 t$ & & & $26 \cdot 9 t$ & & & $2 \cdot 9+$ & \\
\hline Mixed & & $55 \cdot 4 t$ & & & $51 \cdot 8+$ & & & $57 \cdot 2 \dagger$ & \\
\hline \multicolumn{10}{|l|}{ Alcohol consumption } \\
\hline Never & & $100 \cdot 0+$ & & & $4 \cdot 2 \dagger$ & & & $57 \cdot 0+$ & \\
\hline At least once a month & & $0 \cdot 0+$ & & & $95 \cdot 8+$ & & & $43 \cdot 0+$ & \\
\hline Smokers & & $0 \cdot 5+$ & & & $36 \cdot 5 t$ & & & $8 \cdot 2 \dagger$ & \\
\hline
\end{tabular}

PA, physical activity.

a,b,c Mean values within a column with unlike superscript letters were significantly different between the clusters (post hoc Bonferroni testing). Significant differences between the clusters: $+P<0.01$ ( $\chi^{2}$ test).

Table 3 Sociodemographic characteristics of the study population within lifestyle cluster: cross-sectional data, Kiel Obesity Prevention Study, 2004-2006

\begin{tabular}{|c|c|c|c|c|c|c|}
\hline & \multicolumn{2}{|c|}{ Cluster 1} & \multicolumn{2}{|c|}{ Cluster 2} & \multicolumn{2}{|c|}{ Cluster 3} \\
\hline & \multicolumn{2}{|c|}{$\begin{array}{l}\text { Low activity and low-risk } \\
\text { behaviour }(n 740)\end{array}$} & \multicolumn{2}{|c|}{$\begin{array}{c}\text { High media time and high-risk } \\
\text { behaviour }(n \text { 498) }\end{array}$} & \multicolumn{2}{|c|}{$\begin{array}{l}\text { High activity and medium-risk } \\
\text { behaviour ( } n 656)\end{array}$} \\
\hline & Median & IQR & Median & IQR & Median & IQR \\
\hline \multirow[t]{2}{*}{ Age (years) } & $14 \cdot 6^{*}$ & $14 \cdot 2-14 \cdot 9$ & $14 \cdot 9$ & $14 \cdot 5-15 \cdot 5$ & $14 \cdot 6$ & $14 \cdot 3-15 \cdot 1$ \\
\hline & \multicolumn{2}{|c|}{$\%$} & \multicolumn{2}{|c|}{$\%$} & \multicolumn{2}{|c|}{$\%$} \\
\hline $\begin{array}{l}\text { Boys/girls } \\
\text { Educational level }\end{array}$ & \multicolumn{2}{|c|}{$54 \cdot 7 / 45 \cdot 3+$} & \multicolumn{2}{|c|}{$55 \cdot 0 / 45 \cdot 0 t$} & \multicolumn{2}{|c|}{$36 \cdot 4 / 63 \cdot 6 t$} \\
\hline Low & \multicolumn{2}{|c|}{$21 \cdot 1 \dagger$} & \multicolumn{2}{|c|}{$24 \cdot 1+$} & \multicolumn{2}{|c|}{$14 \cdot 3+$} \\
\hline Middle & \multicolumn{2}{|c|}{$40 \cdot 3 t$} & \multicolumn{2}{|c|}{$45 \cdot 8 \dagger$} & \multicolumn{2}{|c|}{$32 \cdot 2 t$} \\
\hline High & \multicolumn{2}{|c|}{$38 \cdot 6+$} & \multicolumn{2}{|c|}{$30 \cdot 1 t$} & \multicolumn{2}{|c|}{$53 \cdot 5 t$} \\
\hline Non-German & \multicolumn{2}{|c|}{$17 \cdot 0 t$} & \multicolumn{2}{|c|}{$8.6+$} & \multicolumn{2}{|c|}{$14 \cdot 2 t$} \\
\hline
\end{tabular}

IQR, interquartile range.

Significant differences between the clusters: ${ }^{\star} P<0.05$ (Kruskal-Wallis test); $\uparrow P<0.05$ ( $\chi^{2}$ test).

All the adolescents in this cluster reported never drinking alcohol, and only $0.5 \%$ smoked regularly.

Cluster 2 was observed in $26 \cdot 3 \%$ of adolescents. Subjects of this cluster showed moderately structured and unstructured PA and high media time. In one-fourth of the adolescents, the dietary pattern was risk-related; more than one-third smoked and almost all consumed alcohol at least once a month.

Of the adolescents, 34.6\% were in cluster 3. This cluster was characterized by high $Z$-scores for structured and unstructured PA combined with low Z-scores for media time. A risk-related dietary pattern was found in $2.9 \%$ of the adolescents of this cluster. There were $8.0 \%$ who smoked and $43.0 \%$ who consumed alcohol at least once a month.

Table 3 shows the sociodemographic characteristics of the clusters. Clusters 1 and 2 had a higher proportion of boys, whereas cluster 3 comprised more girls. The age of the adolescents was highest in cluster 2 . The clusters differed with regard to the distribution of the educational level. In cluster 1 , the proportion of subjects with middle and high educational levels was similar. Cluster 2 contained the highest proportion of adolescents with a middle educational level, whereas most adolescents in cluster 3 had a high educational level.

\section{Associations with overweight}

Table 4 summarizes the associations between the lifestyle clusters and obesity indices as well as blood pressure. Weight was lowest among adolescents in cluster 3. Adolescents of cluster 2 were taller than adolescents of the other clusters. BMI was lowest among adolescents in cluster 1, whereas FM was lowest among adolescents in cluster 2. 
Table 4 Associations between lifestyle clusters and obesity indices and BP of the study population: cross-sectional data, Kiel Obesity Prevention Study, 2004-2006

\begin{tabular}{|c|c|c|c|c|c|c|}
\hline & \multicolumn{2}{|c|}{ Cluster 1} & \multicolumn{2}{|c|}{ Cluster 2} & \multicolumn{2}{|c|}{ Cluster 3} \\
\hline & \multicolumn{2}{|c|}{$\begin{array}{l}\text { Low activity and low-risk } \\
\text { behaviour }(n 740)\end{array}$} & \multicolumn{2}{|c|}{$\begin{array}{l}\text { High media time and high- } \\
\text { risk behaviour ( } n \text { 498) }\end{array}$} & \multicolumn{2}{|c|}{$\begin{array}{c}\text { High activity and medium-risk } \\
\text { behaviour ( } n \text { 656) }\end{array}$} \\
\hline & Median & IQR & Median & IQR & Median & IQR \\
\hline \multicolumn{7}{|l|}{ Parameters of adiposity } \\
\hline Weight (kg) & $58 \cdot 0^{*}$ & $51 \cdot 2-65 \cdot 4$ & $60 \cdot 2$ & $54 \cdot 1-67 \cdot 9$ & $57 \cdot 3$ & $51 \cdot 6-64 \cdot 4$ \\
\hline Height (m) & $1 \cdot 68^{*}$ & $1 \cdot 62-1 \cdot 74$ & 1.69 & $1 \cdot 64-1 \cdot 76$ & 1.68 & $1 \cdot 63-1 \cdot 72$ \\
\hline $\operatorname{BMI}\left(\mathrm{kg} / \mathrm{m}^{2}\right)$ & $20 \cdot 1^{*}$ & $18 \cdot 6-22 \cdot 7$ & $20 \cdot 8$ & $19 \cdot 0-22 \cdot 9$ & $20 \cdot 3$ & $18 \cdot 8-22 \cdot 2$ \\
\hline BMI-SDS & $0 \cdot 19$ & $-0.37-0.90$ & 0.33 & $-0.33-0.94$ & 0.19 & $-0.39-0.77$ \\
\hline FM (\%) & $22 \cdot 3^{*}$ & $16 \cdot 4-28 \cdot 2$ & $21 \cdot 9$ & $15 \cdot 5-27 \cdot 0$ & $23 \cdot 1$ & $17 \cdot 7-28 \cdot 3$ \\
\hline WC $(\mathrm{cm})$ & $69 \cdot 4 t$ & $65 \cdot 4-74 \cdot 5$ & $70 \cdot 8$ & $67 \cdot 0-75 \cdot 5$ & $68 \cdot 8$ & $65 \cdot 0-73 \cdot 0$ \\
\hline Prevalence of overweight (\%) & \multirow{2}{*}{\multicolumn{2}{|c|}{$\begin{array}{r}15 \cdot 5 \\
4 \cdot 9\end{array}$}} & \multicolumn{2}{|c|}{$15 \cdot 7$} & \multicolumn{2}{|r|}{$12 \cdot 3$} \\
\hline Prevalence of obesity (\%) & & & & 8 & & $3 \cdot 5$ \\
\hline \multicolumn{7}{|l|}{ BP } \\
\hline $\mathrm{BP}_{\text {sys }}(\mathrm{mmHg})$ & 120 & $110-130$ & 120 & $110-128$ & 120 & $110-125$ \\
\hline $\mathrm{BP}_{\text {dias }}(\mathrm{mmHg})$ & 72 & $66-80$ & 72 & $68-80$ & 72 & $70-80$ \\
\hline
\end{tabular}

IQR, interquartile range; $\mathrm{BMI}-\mathrm{SDS}$, SD score of $\mathrm{BMI}$; FM, fat mass; WC, waist circumference; $\mathrm{BP}$, blood pressure; $\mathrm{BP}_{\text {sys }}, \mathrm{systolic}_{\mathrm{BP}}$; $\mathrm{BP}_{\text {dias, }}$, diastolic $\mathrm{BP}_{\text {. }}$ Significant differences between the clusters: ${ }^{\star} P<0.05$ ( $\chi^{2}$ test); $+P<0.01$ (Kruskal-Wallis test).

There was no significant difference in the prevalence of overweight and obesity between the clusters with regard to the total study population. However, prevalence tended to be lowest in cluster 3. Regarding boys and girls separately, girls in cluster 3 had a significantly lower prevalence of overweight $(11 \cdot 0 \%)$ than girls in clusters $1(17 \cdot 0 \%)$ and $2(14.3 \% ; P=0 \cdot 017)$. Considering the educational level of the adolescents, no differences in the prevalence of overweight and obesity existed between the clusters.

Blood pressure did not differ between the clusters.

\section{Longitudinal data}

Associations with the development of overweight Table 5 shows the longitudinal data of obesity indices in the subsample by lifestyle cluster identified at the age of 14 years. At T0, the lowest weight and BMI were found among children in cluster 1 . By contrast, WC was lowest in cluster 3. At T1, compared with the other clusters, weight and WC were lowest in cluster 3 .

Adjusted 4-year changes of FM were found to be lowest in cluster 2. Stratifying for gender, these results were confirmed in girls (data not shown).

There was no difference in the prevalence of overweight and obesity between the clusters either at T0 or at T1. However, the 4-year incidence rates of obesity were significantly lower in cluster 3. Sex and educational level had no confounding effect on prevalence and incidence rates.

\section{Discussion}

The aim of the present study was to characterize lifestyle clusters in German adolescents and to analyse their potential association with overweight. Cluster analyses identified three groups of adolescents sharing similar lifestyle behaviour patterns. These clusters varied by sociodemographics and tended to differ in their associations with overweight and obesity. Compared to cluster 3, clusters 1 and 2 tended to have a higher prevalence of overweight and obesity. In addition, the incidence rate of obesity was lowest in cluster 3 .

\section{Lifestyle factors}

Today, participating in PA as well as the use of electronic media has a great role in adolescents' recreational behaviour. German adolescent boys and girls spend 3·5-fold and $2 \cdot 5$-fold time in using electronic media than in exercising and playing sports, respectively ${ }^{(27,28)}$. In addition, $20 \%$ of $11-17$-year-old adolescents in Germany smoke, one-third of the boys and one-quarter of the girls consume alcohol regularly ${ }^{(29)}$. Several studies have shown that modifiable lifestyle factors are interrelated.

The increasing impact of using electronic media is often stated to be one of the main reasons for the supposed lack of PA in adolescents. The so-called 'displacement hypothesis' proposes that sedentary behaviours displace $\mathrm{PA}^{(30)}$. However, controversial findings exist concerning these associations ${ }^{(2,16,31)}$. Correlations between PA and inactivity (high media times) are often small or do not exist. However, in the present study, PA and media time were inversely but weakly correlated $(r=-0.08$ and $-0 \cdot 14$ in boys and girls, respectively). Adolescents with the highest PA (cluster 3) were characterized by the shortest media times. Conversely, adolescents in cluster 1 did not have the longest media time. These findings are in line with the results of te Velde et al. ${ }^{(17)}$ suggesting that certain sedentary behaviours, such as TV viewing and computer use, are not necessarily barriers for PA. One reason for that could be that $\mathrm{PA}$ is executed typically in the afternoon while sedentary behaviours are more prevalent in the evening. 


\section{Lifestyle clusters}

In our study population, we identified three lifestyle patterns. Adolescents with low PA levels (cluster 1) did not show any risk behaviour in terms of alcohol consumption and smoking. By contrast, high media times were combined with poor nutrition and high-risk behaviour in terms of alcohol consumption and smoking (cluster 2). In addition, high activity was correlated with low media time, healthy nutrition together with high alcohol consumption and smoking (cluster 3). Strictly speaking, none of these clusters was considered to be absolute healthy.

te Velde et $a l .{ }^{(17)}$ characterized a so-called healthy behaviour pattern in 8-13-year-old children. However, the authors did not consider risk behaviour in the analysis and labelling of the cluster was based on low time spent on TV viewing during dinner and during the day and low computer use combined with high PA. Associations with risk behaviour stayed unclear. Boone-Heinonen et al. ${ }^{(32)}$ performed a cluster analysis including risk behaviour in 11-21-year-old US adolescents. They found that high smoking prevalence was associated with junk food consumption in boys and food restriction in girls. In contrast to our findings, the sedentary behaviour cluster was characterized by low alcohol consumption and low smoking prevalence.

The clusters identified in our study differed by sociodemographic characteristics. Adolescents of the 'high media time and high-risk behaviour' cluster were older than adolescents of the other clusters. Since alcohol and cigarette consumption increase with age in adolescents ${ }^{(29)}$, this could explain the high consumption in this cluster. In contrast to te Velde et $a l^{(17)}$, different behavioural patterns for boys and girls could not be found in the present study. Identified clusters had identical characteristics for both sexes.

\section{Associations with overweight}

We found no clear associations between lifestyle clusters and overweight and obesity in our study population. Prevalence did not significantly differ between the clusters but tended to be lowest in cluster 3. However, 4-year incidence of obesity was significantly lowest in cluster 3 and highest in cluster 1 , suggesting that low activity plays a major role in the development of childhood obesity.

While previous studies used cluster analyses to explore patterns of these behaviours ${ }^{(15,33,34)}$, few analyses examined the association of lifestyle clusters with overweight ${ }^{(17,32)}$. te Velde et $a l .{ }^{(17)}$ found the lowest prevalence of overweight in the healthy behaviour cluster (characterized by high PA and low TV viewing and computer use) supporting our findings. In the study of Boone-Heinonen et $a l^{(32)}$, the cluster characterized by participation in many school clubs and sports had the lowest risk of overweight.

\section{Study strength and limitations}

A strength of the present study is cluster analysis instead of characterization of isolated individual behaviours. 
In addition, the study provides longitudinal data to test associations between lifestyle clusters and weight change.

However, some limitations should be addressed. Since we assumed that adolescents of the subsample had retained their lifestyle behaviour pattern over a 4-year time period from 10-14 years of age, we cannot rule out some changes in lifestyle behaviours over that time. It would have been more precise to repeat cluster analysis, at the age of 10 and 14 years, and to account for changes in lifestyle behaviours. However, previous studies have shown that PA and sedentary behaviour track from childhood to adolescence ${ }^{(35,36)}$.

While the weakness of self-reported measures of lifestyle behaviours of PA and nutrition is well known ${ }^{(37,38)}$, our activity and nutrition questions were based on questionnaires demonstrated to be valid ${ }^{(21,22)}$ and reliable ${ }^{(20)}$ and have been shown to be important predictors of obesity ${ }^{(39)}$. Only one typical sedentary behaviour, i.e. time spent in TV viewing and computer use, was assessed. However, adolescents engage in a wide range of activities that have not often been assessed, e.g. doing homework, reading, personal care and social interactions ${ }^{(40)}$. Using electronic media is the most popular sedentary recreational behaviour among German adolescents ${ }^{(27)}$

For a large proportion of respondents, data in terms of lifestyle behaviour were missing (40.8\%), because on the one hand, the questionnaire was enlarged during the examination. Thus, items required for this analysis, such as commuting mode to school and unstructured PA, were not available for $76 \cdot 2 \%$ of adolescents measured in 2004 . On the other hand, incomplete questionnaires were returned. In comparison with the excluded adolescents, the proportion of adolescents with a high educational level was lower and the frequency of non-Germans was higher in the study population, but the distribution of gender, prevalence of overweight and obesity and participation in structured PA were similar. Since cluster solution was reconstructed in a random sample, our results and conclusions would have been comparable when including a larger sample. Nevertheless, dropout bias cannot be completely ruled out.

\section{Conclusions}

Explicit healthy lifestyles do not exist among adolescents of the KOPS. An active lifestyle pattern reduces the incidence of obesity. Our findings provide strong support for targeted interventions that account for diversity of lifestyles and address-specific lifestyle clusters. This strategy seems to be more promising than one-for-all programmes.

\section{Acknowledgements}

Sources of funding: KOPS is supported by grants from Deutsche Forschungsgemeinschaft (DFG Mü 5-1, 5-2, 5-3,
5-5), 'Kompetenznetz Adipositas (Competence Network on Obesity)' funded by the Federal Ministry of Education and Research (FKZ: 01GI0821), Wirtschaftliche Vereinigung Zucker, Precon and WCRF. The present study has been facilitated by the EU-funded HOPE project: Health Promotion through Obesity Prevention across Europe (the Commission of the European Communities, SP5A-CT-2006-044128). The study does not necessarily reflect the Commission's views and in no way anticipates the Commission's future policy in this area. Conflict of interest declaration: The authors have no conflict of interest. Authorship responsibilities: B.L. had the original idea, conducted the statistical analyses, did the interpretation of the data and wrote the manuscript. B.L., D.L., M.J. and J.S. acquired the data. S.P.-D. and M.J.M. supervised the study, did the interpretation of the data and wrote the paper. All authors discussed the data and approved the final version of the paper.

\section{References}

1. Hancox RJ, Milne BJ \& Poulton R (2004) Association between child and adolescent television viewing and adult health: a longitudinal birth cohort study. Lancet $\mathbf{3 6 4}$, 257-262.

2. Marshall SJ, Biddle SJ, Gorely T et al. (2004) Relationships between media use, body fatness and physical activity in children and youth: a meta-analysis. Int J Obes Relat Metab Disord 28, 1238-1246.

3. Kleiser C, Schaffrath Rosario A, Mensink GB et al. (2009) Potential determinants of obesity among children and adolescents in Germany: results from the cross-sectional KiGGS Study. BMC Public Health 9, 46.

4. Ludwig DS, Peterson KE \& Gortmaker SL (2001) Relation between consumption of sugar-sweetened drinks and childhood obesity: a prospective, observational analysis. Lancet 357, 505-508.

5. James J, Thomas P, Cavan D et al. (2004) Preventing childhood obesity by reducing consumption of carbonated drinks: cluster randomised controlled trial. BMJ 328, 1237.

6. Malik VS, Schulze MB \& Hu FB (2006) Intake of sugarsweetened beverages and weight gain: a systematic review. Am J Clin Nutr 84, 274-288.

7. Kubik MY, Lytle LA \& Story M (2005) Schoolwide food practices are associated with body mass index in middle school students. Arch Pediatr Adolesc Med 159, 1111-1114.

8. Molnar D \& Livingstone B (2000) Physical activity in relation to overweight and obesity in children and adolescents. Eur J Pediatr 159, Suppl. 1, S45-S55.

9. Kimm SY, Glynn NW, Obarzanek E et al. (2005) Relation between the changes in physical activity and body-mass index during adolescence: a multicentre longitudinal study. Lancet 366, 301-307.

10. Janssen I, Katzmarzyk PT, Boyce WF et al. (2005) Comparison of overweight and obesity prevalence in school-aged youth from 34 countries and their relationships with physical activity and dietary patterns. Obes Rev 6, 123-132.

11. Chen X, Beydoun MA \& Wang Y (2008) Is sleep duration associated with childhood obesity? A systematic review and meta-analysis. Obesity 16, 265-274.

12. Hitze B, Bosy-Westphal A, Bielfeldt F et al. (2009) Determinants and impact of sleep duration in children and adolescents: data of the Kiel Obesity Prevention Study. Eur J Clin Nutr 63, 739-746. 
13. Gorely T, Marshall SJ \& Biddle SJ (2004) Couch kids: correlates of television viewing among youth. Int J Behav Med 11, 152-163.

14. van den Bulck J (2000) Is television bad for your health? Behavior and body image of the adolescent "Couch Potato". J Youth Adolesc 29, 273-288.

15. Nelson MC, Gordon-Larsen P, Adair LS et al. (2005) Adolescent physical activity and sedentary behavior: patterning and long-term maintenance. Am J Prev Med 28, 259-266.

16. Biddle SJ, Gorely T, Marshall SJ et al. (2004) Physical activity and sedentary behaviours in youth: issues and controversies. J $R$ Soc Health 124, 29-33.

17. te Velde SJ, De Bourdeaudhuij I, Thorsdottir I et al. (2007) Patterns in sedentary and exercise behaviors and associations with overweight in 9-14-year-old boys and girls - a cross-sectional study. BMC Public Health 7, 16.

18. Müller MJ, Asbeck I, Mast M et al. (2001) Prevention of obesity-more than an intention. Concept and first results of the Kiel Obesity Prevention Study (KOPS). Int J Obes Relat Metab Disord 25, Suppl. 1, S66-S74.

19. Danielzik S, Pust S, Landsberg B et al. (2005) First lessons from the Kiel Obesity Prevention Study (KOPS). Int J Obes 29, Suppl. 2, S78-S83.

20. Landsberg B, Plachta-Danielzik S, Much D et al. (2008) Associations between active commuting to school, fat mass and lifestyle factors in adolescents: the Kiel Obesity Prevention Study (KOPS). Eur J Clin Nutr 62, 739-747.

21. Grund A, Krause H, Siewers M et al. (2001) Is TV viewing an index of physical activity and fitness in overweight and normal weight children? Public Health Nutr 4, 1245-1251.

22. Mast M, Kortzinger I \& Müller MJ (1998) Ernährungsverhalten und Ernährungszustand 5-7-jähriger Kinder in Kiel. Akt Ernäbr Med 23, 282-288.

23. Plachta-Danielzik S, Landsberg $\mathrm{B}$, Johannsen $\mathrm{M}$ et al. (2008) Association of different obesity indices with blood pressure and blood lipids in children and adolescents. Br J Nutr 100, 208-218.

24. Cole TJ, Bellizzi MC, Flegal KM et al. (2000) Establishing a standard definition for child overweight and obesity worldwide: international survey. BMJ 320, 1240-1243.

25. Kromeyer-Hauschild K, Wabitsch M, Kunze D et al. (2001) Perzentile für den body-mass-index für das Kindes- und Jugendalter unter Heranziehung verschiedener deutscher Stichproben. Monatsschr Kinderbeilkd 149, 807-818.

26. Everitt BS, Landau S \& Leese M (2001) Cluster Analysis, 4th ed. London: Hodder Arnold.

27. Lampert T, Sygusch R \& Schlack R (2007) Use of electronic media in adolescence. Results of the German Health Interview and Examination Survey for Children and
Adolescents (KiGGS). Bundesgesundheitsblatt Gesundbeitsforschung Gesundheitsschutz 50, 643-652.

28. Lampert T, Mensink GB, Romahn N et al. (2007) Physical activity among children and adolescents in Germany. Results of the German Health Interview and Examination Survey for Children and Adolescents (KiGGS). Bundesgesundheitsblatt Gesundheitsforschung Gesundheitsschutz 50, 634-642.

29. Lampert $\mathrm{T} \&$ Thamm M (2007) Consumption of tobacco, alcohol and drugs among adolescents in Germany. Results of the German Health Interview and Examination Survey for Children and Adolescents (KiGGS). Bundesgesundheitsblatt Gesundheitsforschung Gesundheitsschutz 50, 600-608.

30. Mutz DC, Roberts DF \& van Vuuren DP (1993) Reconsidering the displacement hypothesis: television's influence on children's time use. Communic Res 20, 51-75.

31. Sallis JF, Prochaska JJ \& Taylor WC (2000) A review of correlates of physical activity of children and adolescents. Med Sci Sports Exerc 32, 963-975.

32. Boone-Heinonen J, Gordon-Larsen P \& Adair LS (2008) Obesogenic clusters: multidimensional adolescent obesityrelated behaviors in the US. Ann Behav Med 36, 217-230.

33. Gorely T, Marshall SJ, Biddle SJ et al. (2007) Patterns of sedentary behaviour and physical activity among adolescents in the United Kingdom: Project STIL. J Behav Med 30, 521-531.

34. Hulshof KF, Wedel M, Lowik MR et al. (1992) Clustering of dietary variables and other lifestyle factors (Dutch Nutritional Surveillance System). J Epidemiol Community Health 46, 417-424.

35. Janz KF, Dawson JD \& Mahoney LT (2000) Tracking physical fitness and physical activity from childhood to adolescence: the muscatine study. Med Sci Sports Exerc 32, 1250-1257.

36. Kristensen PL, Moller NC, Korsholm L et al. (2008) Tracking of objectively measured physical activity from childhood to adolescence: the European youth heart study. Scand J Med Sci Sports 18, 171-178.

37. Sirard JR \& Pate RR (2001) Physical activity assessment in children and adolescents. Sports Med 31, 439-454.

38. Willett W (1998) Overview of Nutritional Epidemiology. Nutritional Epidemiology, 2nd ed. New York: Oxford University Press, pp. 3-17.

39. Plachta-Danielzik S, Landsberg B, Lange D et al. (2010) Determinants of prevalence and incidence of overweight in children and adolescents. Public Health Nutr (Epublication ahead of Print version).

40. Jago R, Anderson CB, Baranowski $\mathrm{T}$ et al. (2005) Adolescent patterns of physical activity differences by gender, day, and time of day. Am J Prev Med 28, 447-452. 